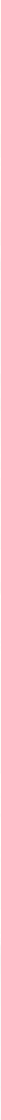




\title{
Procesos de admisión para la educación superior en Venezuela. Análisis de aspectos teóricos y éticos
}

\author{
Amalio SARCo \\ Maestro normalista rural de la Escuela Normal Luis Alejandro Alvarado, director de la \\ escuela primaria Instituto de Mejoramiento Profesional del Magisterio UPEL. Licenciado en \\ Educación con mención en la Planificación Educativa UCV. Master en Educación superior \\ UCV. Doctorando en Educación UCV. \\ Profesor asociado a la Escuela de Educación ucv. Gerente del Desarrollo Docente y \\ Estudiantil adscrito al vicerrectorado académico de la UCV. Jefe del Departamento de \\ Estadística e Informática de la Escuela de Educación ucv. Coordinador del Proceso \\ Nacional de Admisión en la Oficina de Planificación del Sector Universitario OPSU del \\ Consejo Nacional de Universidades CNU.
}

\section{Resumen}

El proceso de admisión a la educación superior fue desarrollado por añadidura hasta convertirse en un "conjunto de servicios que acompañaban al estudiante en su tránsito desde la educación media hasta la superior”. Los aspirantes recibían atención permanente y eran evaluados en aptitudes académicas, conocimientos específicos, gustos e intereses y despistaje vocacional, todos los criterios de selección eran auditables. A partir del 2008 el Gobierno Nacional impuso el Modelo Multivariable para la selección, lo que determinó que el 39\% de la información resultara inauditable. En el 2015 se introdujeron nuevos procedimientos que hicieron que el $50 \%$ de la información del índice fuera inauditable. Las autoridades universitarias han propuesto alternativas para el ingreso como la asistencia académica con documentos que aún esperan para ser evaluados.

Palabras clave

Ingreso; admisión; educación superior; criterios de selección; transparencia; auditabilidad. 


\title{
Admission processes to higher education in Venezuela: An analysis of ethical and theoretical aspects
}

\begin{abstract}
The process of admission to higher education in Venezuela had been developed by aggregation until it turned into a "group of services that accompanied the students in their way from high school to university". Applicants received permanent attention, and their academic aptitudes, specific types of knowledge, interests and vocational orientation were evaluated; all selection criteria were subject of audit. In 2008, the national government imposed the multivariable model for the selection process, which determined that $39 \%$ of information did not require audit. Later, in 2015, the government introduced new procedures which make that from now on $50 \%$ of index information does not receive examination. University authorities have proposed alternatives to the admission based on academic assistance, but they are documents waiting to be evaluated.
\end{abstract}

Key words

Admission, Higher education, Selection criteria, Transparency, Audit.

\section{Os processos de admissão para o ensino superior na Venezuela. Análise dos aspetos teóricos e éticos}

Sumário

O processo de admissão para o ensino superior foi construido como uma agregação até se tornar num "Conjunto de Serviços que acompanhavam aos estudantes no trânsito do ensino médio ao superior”. Os aspirantes recebiam atenção permanente e eram avaliados em suas aptidões acadêmicas, conhecimentos específicos, gostos, intereses e vocações; tudos os critérios de seleção foram auditáveis. A partir do ano 2008, o Governo Nacional impôs o Modelo Multivariável para as seleções, o que determinaria que um $39 \%$ da informação não era auditável. No ano 2015 foram introduzidos novos procedimentos que fizeram que um $50 \%$ de informação do índice não fora auditável. As autoridades universitárias propuseram alternativas para o ingresso como a assitência acadêmica com documentos que esperam ser avaliados.

Palavras-chave

Ingresso; admissão; ensino superior; critérios de seleção; transparência; auditabilidade. 


\section{Antecedentes}

El 23 de enero de 1958 cae el gobierno del general Marcos Evangelista Pérez Jiménez, esta dictadura, que inició en el año 1947 con el derrocamiento del gobierno democrático del escritor Rómulo Gallegos, llega a su fin y el gobierno de transición es encabezado por el vicealmirante Wolfgang Larrazábal Ugueto. La llegada de la democracia trajo consigo el desarrollo de políticas para el acercamiento de los servicios a las capas populares, y en el caso de la educación, se emprende un ambicioso programa de construcción de locales escolares para ampliar la cobertura inicial de la educación primaria (EP), y posteriormente la secundaria. En paralelo, con la ampliación de la Escuela Normal para la formación de los maestros de primaria, y de los institutos pedagógicos para la formación de los profesores de educación media (EM), se daba respuesta a los requerimientos del personal docente de estos niveles educativos.

Durante la dictadura y los primeros años de la democracia (Junta de gobierno 1958-1959 y gobierno de Rómulo Betancourt 1959-1964), la continuación de los estudios requería que, al concluir la primaria, los aspirantes se registraran en las instituciones secundarias, donde continuaban sus estudios para participar en un proceso de selección, y solo en caso de ser admitido, el aspirante podía inscribirse para seguir su formación escolar. De estos exámenes de admisión no se disponen registros, a la distancia suponemos que el proceso se regía por el Numerus Clausus, es decir, se establecía la capacidad de atención del instituto, se ordenaba la demanda con base en las puntuaciones alcanzadas, y se seleccionaba esa cantidad de aspirantes para iniciar la formación en EM. De manera progresiva, estos exámenes fueron suprimidos, y apareció en Venezuela el fenómeno que se conoció como la "Masificación de la Enseñanza" tanto en EP como en EM.

Hasta ese momento, el ingreso a la educación superior (ES) era un proceso rústico, consistía en obtener el título de bachiller y esperar a que las instituciones de educación superior (IES) hicieran el llamado a la inscripción, en el caso de la Universidad Central de Venezuela (UCV), que históricamente ha sido la institución de mayor demanda, los aspirantes acudían al llamado desde la noche anterior y se enfilaban frente a los nichos del Aula Magna, donde se instalaban las taquillas por escuelas: usted seleccionaba la carrera de su preferencia, se incorporaba a la fila correspondiente y el orden de llegada fungía como la variable ordenadora para la asignación de los cupos, el aspirante sabía que tenía lugar si al llegar encontraba la taquilla abierta, estas cerraban al agotarse las plazas disponibles. De ser este el caso existía el cambio de opción, y el criterio para seleccionar la segunda opción regularmente consistía en que la fila no estuviera tan larga. Esta práctica funcionó hasta entrados los años setenta, hacia finales 
de la década, por efectos de la masificación de los niveles educativos anteriores, la demanda de la ES ya superaba abiertamente la oferta de plazas, por lo que se crearon en las IEs los "Comités de Bachilleres sin Cupo", los cuales estuvieron muy activos en sus protestas en lo interno de las instituciones. En algunas de ellas, las manifestaciones eran constantes y ocurrían actividades ordinarias en las distintas carreras, que se veían interrumpidas con regularidad, ocasionando la pérdida de los lapsos académicos, fueran trimestres, semestres o años.

\section{Los modelos de admisión e ingreso utilizados por el Consejo Nacional de Universidades (CNU) a partir del año 1973}

En 1973 el CNU, con el fin de conocer las tendencias y la cuantía de la demanda por Es, creó una comisión de investigación integrada por docentes de diferentes universidades nacionales, que decidió la formulación de un cuestionario aplicado como encuesta para recabar información personal, académica y socioeconómica de cada posible demandante; se propuso hacer un censo de población (CNU, 1973). Este cuestionario, llenado por todos los estudiantes del último año de EM y buena parte de los bachilleres que se mantenían pendientes para el ingreso a la ES, fue llamado "Preinscripción Nacional", y los participantes entendieron que el paso siguiente era la inscripción. Los Comités de Bachilleres sin Cupo, que hasta ese momento accionaban sus protestas en lo interno de las universidades, ahora lo hacían en las calles, el problema institucional se había vuelto nacional. De manera que el CNU se vio obligado, ante la presión social ejercida por los encuestados, a convertir una comisión de investigación en un equipo técnico de selección y asignación de plazas en la educación superior, para lo cual, se solicitó la capacidad de atención a las diferentes instituciones (cupos), y a los aspirantes las calificaciones obtenidas durante la EM, para ordenarlos con base en un requisito que todos pudieran satisfacer (Sarco y Bonucci, 1994). Esta solicitud a los aspirantes resultó afortunada, ya que con el tiempo se pudo conocer que los antecedentes académicos del aspirante, como el promedio de calificaciones de EM, es de forma aislada el mejor predictor del rendimiento académico en la ES, el que alcanza la mayor correlación con el récord académico, o promedio de calificaciones finales, al culminar el primer año de la carrera.

Las IES, a su vez, ofrecieron las plazas existentes a través de esta nueva forma de ingreso, librándose de los reclamos que hasta ese momento no les permitían el desarrollo de sus actividades cotidianas, las quejas y protestas ahora serían atendidas por el CNU a través de la Oficina de Planificación del Sector Universitario (OPSU). Tiempo después aparecerían de nuevo los llamados mecanismos institucionales de ingreso, administrados por las facultades o escuelas.

De esta manera, el Proceso Nacional de Admisión (PNA) surge como una reivindicación del movimiento estudiantil del momento, fue creado en el año 1973 de forma experimental (Sarco y Bonucci, 1994), con la intención de aplicar una metodología de asignación a las IES oficiales (públicas) del país. Es a partir de este momento que se inician en Venezuela los estudios base para 
la construcción de las Pruebas de Aptitud Académica (PAA), cuyas primeras versiones se aplicaron en el año 1978, dos años más tarde comenzaron a aplicarse las versiones definitivas con carácter voluntario, desde 1980 hasta 1983. Su aplicación se hizo obligatoria desde 1984 para toda la población que aspiraba ingresar al subsistema de educación superior. Esto permitió al Estado tener una estadística nacional de la demanda de aspirantes cada año.

El PNA para la ES en Venezuela fue coordinado por la OPSU por decisión del CNU. La mayoría de las IES oficiales del país aceptaron la asignación de un porcentaje importante de sus estudiantes a través de este procedimiento, cuya principal ventaja era que permitía la postulación de cada candidato a la educación superior desde su propia región o zona de residencia, aunque fuera lejana a la IES a la que decidía aplicar y podía hacerla desde su pupitre. En la UCV todas las facultades atendieron el ingreso directo de los estudiantes por esta vía.

De forma progresiva el PNA se constituyó como un conjunto de servicios que acompañaba al estudiante en el tránsito desde la EM, diversificada y profesional, hasta la ES. Se publicó anualmente la información sobre oportunidades de estudio, se realizaron jornadas de difusión en las entidades federales, se evaluó el desarrollo verbal y numérico a través de la PAA, se publicaron diferentes manuales de procedimiento para orientar a los usuarios, se desarrollaron pruebas de conocimientos específicos por asignatura de EM -que resultaron muy valiosas para definir el ingreso a la Universidad Pedagógica Experimental Libertador- y se realizó la evaluación vocacional, que fue el último servicio desarrollado. La atención de la población demandante se hizo permanente, de manera que los reclamos que pudieran suscitarse serían atendidos como parte del proceso (Sarco, 2003).

Descripción de las fases del Proceso Nacional de Admisión (PNA)

- Presentación de la Prueba Nacional de Exploración Vocacional: dirigida a los aspirantes en el primer año de la EM diversificada y profesional. Se ofreció con carácter optativo y su objetivo fue orientar al aspirante en el paso posterior: el registro y postulación a la ES y la selección de carreras e instituciones. El reporte le indicaba al aspirante los grupos de carreras en los cuales 
se preveía que podría tener un mayor desarrollo de sus potencialidades, y en cuáles de ellas podrían favorecerlo sus gustos e intereses. Se le ofrecían en total tres grupos de carreras, donde era el primer grupo el que contenía las más convenientes para el candidato.

- Aplicación de la Prueba de Aptitud Académica (PAA): para explorar las habilidades numéricas y la comprensión lectora de los aspirantes, consistía en la realización de una jornada nacional de aplicación que se realizaba un día sábado a las ocho de la mañana en todas las IES y en algunas instalaciones de EM.

- Jornadas informativas y de orientación sobre oportunidades de estudio en las IES: organizadas en cada entidad federal del país, su objetivo era informar al aspirante sobre las oportunidades de estudio que las IES ofrecen en cada región, e instruirle sobre cómo conducirse en cada una de las fases siguientes del PNA, especialmente la que tiene que ver con el registro y la postulación.

- Registro y postulación para la asignación nacional: se realizaba anualmente y constituía un paso que debían cumplir, con obligatoriedad, todos aquellos aspirantes que deseaban ingresar al subsistema de educación superior en cualquiera de sus instituciones. Se atendía a los estudiantes del último año de EM diversificada y profesional, y a los aspirantes que ya habían egresado de este nivel educativo y deseaban continuar concursando para ingresar al superior.

- Entrega de resultados de la PAA y modificación de datos o postulaciones: los aspirantes recibían el reporte de información individual que contenía su índice académico, con el que podían conocer sus posibilidades de competir por la asignación de un cupo en las IES y las carreras de su preferencia, ya que le señalaban los índices académicos referenciales, representados por el índice que obtuvo el último aspirante que ingresó el año anterior a cada carrera. De esta manera podían hacer ajustes que les permitieran convertirse en mejores candidatos para el ingreso. Se informaba sobre la preasignación de becas OPSU para los aspirantes pertenecientes al nivel socioeconómico "muy pobre", quienes, de ser asignados, obtenían beca de estudios. 
- Asignación nacional: era un proceso totalmente automatizado mediante el cual se asignaba a los aspirantes de acuerdo a las carreras, a las instituciones seleccionadas y a la disponibilidad de cupos reportados por las IES oficiales al CNU. Los expedientes de los usuarios estaban ordenados en un área de once dígitos en la cual se compilaba la información individual correspondiente a los criterios de asignación establecidos de manera progresiva: índice académico, año de graduación, regionalización y nivel socioeconómico. Durante todo el proceso se realizaba una atención permanente al aspirante a través de las oficinas de coordinación ubicadas en las capitales de las diferentes entidades federales del país.

Los criterios de asignación y el cálculo del índice académico en el Proceso Nacional de Admisión

La metodología de asignación de aspirantes utilizada por la OPSU sufrió algunas modificaciones a lo largo de su implementación, sin embargo, hasta el 2007 se basó en los siguientes criterios, que fueron aplicados en el orden que se describen a continuación (Sarco y Fuenmayor, 2004):

Figura 1

Criterios de Asignación del PNA
$\diamond$ Índice académico
$\diamond$ Año de graduación
$\diamond$ Regionalización
$\diamond$ Nivel socioeconómico

- Índice académico: se calculaba el promedio de notas del aspirante, el cual se obtenía considerando sus calificaciones durante los últimos tres años de estudio en la educación básica y el primero en la educación media, diversificada y profesional, esta transformación se efectuaba por separado a los egresados del sector público y a los del privado. El 60\% de este puntaje transformado se incorporaba al índice académico. El 40\% faltante se obtenía del desempeño en la prueba de aptitud académica, constituida por una subprueba de razonamiento matemático (20\%) y otra de comprensión de lectura (20\%). Este era el criterio más importante para la asignación, ofreciéndose prioridad a quienes obtuvieran los índices más altos. Se expresaba con seis dígitos.

- Año de graduación: informaba el tiempo transcurrido desde el momento en que el aspirante obtuvo el título de educación media. En el PNA los aspirantes eran clasificados en estudiantes de EM, a los que se 
asignaba el 9, a los graduados el año anterior se les asignaba un 8, y así sucesivamente (Sarco y Fuenmayor, 2004). Se ofrecía prioridad de asignación a los aspirantes graduados en el mismo año de su solicitud, estableciéndose una jerarquía desde el año más inmediato hasta el menos reciente. Se expresaba con un digito del nueve "9" al cero "0" y era el segundo criterio en importancia.

- Regionalización: se expresaba con un dígito y se refería a la procedencia geográfica según la región administrativa donde estudia o estudió el aspirante la EM. Este era el tercer criterio de asignación, y se ofrecía prioridad a los aspirantes que residían en zonas cercanas a las IES para las que aplicaban su primera opción. Se otorgaba preferencia a los aspirantes que seleccionaban carreras dentro de la región donde obtuvieron el título de educación media utilizando unos " 1 " y ceros " 0 ". La condición de región se consideraba igualmente para la transformación del puntaje de desempeño en la prueba, comparando a los aspirantes entre los de su propia región, al utilizar para ello la media aritmética y la desviación típica de su región respectiva. Este procedimiento se realizaba con la intención de nivelar las diferencias que se observaban, respecto al nivel académico, entre los estudiantes de las regiones más avanzadas y los de otras más deprimidas. El proceso se conoce como "comparación con sus pares".

- Nivel socioeconómico: se determinaba a través de la metodología conocida como Graffar, a la cual la OPSU efectuó algunas modificaciones en 1999. Los aspirantes eran clasificados en cinco niveles a partir de los datos suministrados en un cuestionario que se aplicaba como encuesta: alto, medio alto, medio bajo, obrero y muy pobre (Sarco y Fuenmayor, 2004). Por ser el último criterio de asignación, se ofrecía prioridad a los jóvenes procedentes de hogares en condición de pobreza. Cada pregunta ofrecía cinco alternativas de respuesta, quienes respondían con la alternativa uno en todas las preguntas estaban en las mejores condiciones socioeconómicas, y quienes respondían con la alternativa cinco estaban en las peores condiciones. La suma del puntaje alcanzado por el aspirante se expresaba con dos dígitos.

- Para el ordenamiento los aspirantes se clasificaban en tres grupos en relación con su índice académico (IA): al primer grupo, con IA mayor o igual a 60,000 se asignaba el tres como primer digito; el segundo grupo, de índices mayores a 45,000 pero menores de 60,000 su primer digito era un dos; y se iniciaba con un uno al grupo de los índices menores a 45,000. La información referente a los criterios de asignación se disponía en diferente orden según el grupo al que correspondía el índice académico, fuera para la formación del área o para el sorteo, como se muestra en la siguiente figura. 


\begin{tabular}{|c|c|c|c|c|c|c|c|c|}
\hline \multicolumn{9}{|l|}{$\mid A>60,000$} \\
\hline & No. Dígitos & 3 & \multicolumn{3}{|c|}{ Índice Académico } & AG & CR & SE \\
\hline \multicolumn{9}{|c|}{$45,000<\mathrm{IA}<60,000$} \\
\hline & \multirow[t]{2}{*}{ No. Dígitos } & 1 & 1 & \multicolumn{3}{|c|}{6} & 1 & 1 \\
\hline & & 2 & AG & \multicolumn{3}{|c|}{ Índice Académico } & CR & SE \\
\hline \multicolumn{9}{|l|}{ IA $<45,000$} \\
\hline & \multirow[t]{2}{*}{ No. Dígitos } & 1 & 1 & 1 & \multicolumn{3}{|c|}{6} & 2 \\
\hline & & 1 & SE & AG & \multicolumn{3}{|c|}{ Índice Académico } & SE \\
\hline
\end{tabular}

Creación del Área de 11 dígitos para compilar la información sobre criterios de asignación y ordenar la demanda.

El ordenamiento de la demanda, en sentido estricto de mayor a menor, se hacía con base en este número de once dígitos que condensaba toda la información sobre los criterios de selección. De toda esta información se podían hacer verificaciones con base en los expedientes individuales.

Una vez ordenada la demanda y organizada la tabla de cupos donde corrían los programas de asignación, se consideraba un expediente a la vez, con las opciones de carrera en el orden en el que el aspirante las había seleccionado, hasta finalizar el último expediente de la fila. Como resultado, se obtenían las listas de asignados por carrera en las cantidades establecidas por las instituciones, la lista de aspirantes no asignados, en el orden establecido por el área de once dígitos, y las tablas de cupos sobrantes; toda la información se suministraba a través de las oficinas de atención al público. Generalmente se realizaba un proceso complementario para distribuir los cupos sobrantes entre los bachilleres no asignados que solicitaban a través de una planilla.

Figura 3

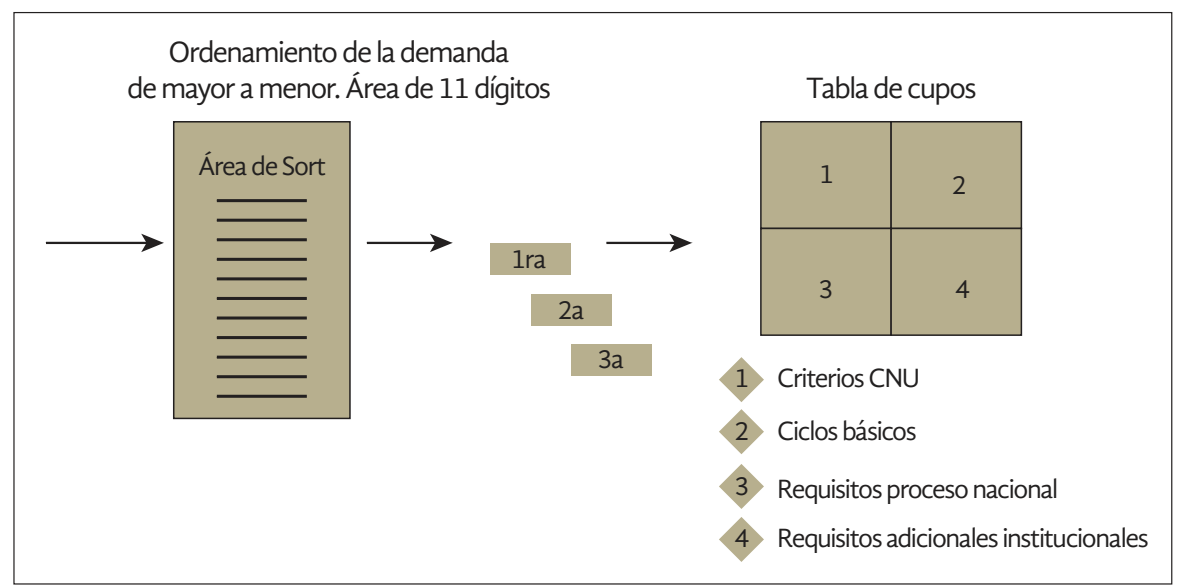




\section{El Proceso Nacional de Ingreso a la educación superior (PNI). Eliminación de la Prueba de Aptitud Académica. Nuevas formas de uso de las variables}

En el año 2007, el CNU decidió eliminar el PNA de la educación superior en Venezuela y establecer el Proceso Nacional de Ingreso (PNI), descartando el uso de la PAA y el índice académico tal cual se calculaba. De esta manera se desechaba el uso de los puntajes de la PAA, que fueron desarrollados en el país por técnicos graduados en nuestras universidades, considerados como el segundo mejor predictor del éxito en la ES en forma aislada; además se evitaba la conformación del índice académico con el promedio de notas que constituye el mejor predictor del éxito en general, según los reportes de diferentes investigaciones (Menéndez, 2000). Se evitó de esta manera conocer el desarrollo de nuestros bachilleres en el uso de los lenguajes de la palabra y los números, además de que el país utilizara la combinación de los dos mejores predictores del éxito para seleccionar los candidatos a estudiar en las IES.

La lógica difusa y los conjuntos borrosos son propuestos por OPSU como base del nuevo modelo de admisión

A finales del 2007 era un hecho la eliminación de la PAA sin que estudios previos justificaran esta medida, y un técnico de oPSU fue el encargado de diseñar el nuevo modelo de selección para el ingreso a la educación superior, la proposición elaborada se basó en la lógica difusa y en el uso de los conjuntos borrosos (Rojas, 2008). Sin restarle mérito a las contribuciones al desarrollo tecnológico que estas construcciones teórico matemáticas han hecho en diferentes sectores de la economía, las universidades nos opusimos a esta proposición por la connotación que tienen estos términos en el lenguaje vulgar culto, y preguntamos cómo podría explicársele, satisfactoriamente, a los padres de un bachiller que no había quedado seleccionado para estudiar en la universidad que se le había aplicado un modelo basado en la lógica difusa, en el cual se utilizaban los conjuntos borrosos por los que el bachiller había sido rechazado. El modelo entonces paso a denominarse Índice Multivariado para el ingreso a la Es. Los representantes de las universidades mantuvimos el rechazo al índice porque casi el $40 \%$ de la información que lo constituía era inauditable, no había manera de verificarla. Además de lo discutible que resultaba llamar Multivariable a un proceso en el que el $97.5 \%$ de la información dependía de las calificaciones que exhiba cada aspirante.

\section{El Modelo Multivariado para el ingreso a la educación superior}

Según esta proposición (CNU, 2009) el nuevo índice estaría conformado por el promedio general de notas en un $48.75 \%$, y por el promedio de notas específico por área de conocimiento en un $48.75 \%$, quedando un $2.5 \%$ disponible para consideraciones como la participación en eventos anteriores sin haber obtenido lugar en esos procesos (González, 2008). 
Figura 4

Variables

\begin{tabular}{|c|c|c|}
\hline Condición socio-educativa: & & \\
\hline Promedio global de notas: $30 \%$ & 29.25 & \\
\hline $\begin{array}{c}\text { Comparación del promedio global de notas con la mediana } \\
\text { del grupo de referencia del plantel: } 20 \%\end{array}$ & 19.50 & \\
\hline Promedio de notas por área del alumno: 30\% & 29.25 & \\
\hline \multirow[t]{2}{*}{$\begin{array}{l}\text { Comparación del Promedio de notas por área de } \\
\text { conocimiento con el grupo de referencia del plantel: 20\% }\end{array}$} & 19.50 & \\
\hline & & 97.50 \\
\hline \multicolumn{3}{|l|}{ Otros factores: } \\
\hline Región: 12\% & 0.30 & \\
\hline Participación previa en proceso CNU: 62.86\% & 1.57 & \\
\hline \multirow[t]{2}{*}{ Asignación previa a cupo por el CNU: 25.14\% } & 0.63 & \\
\hline & & 2.50 \\
\hline
\end{tabular}

Fuente: Gobierno Bolivariano de Venezuela, Ministerio del Poder Popular para la Educación Universitaria, Consejo Nacional de Universidades, Oficina de Planificación del Sector Universitario, Programa Nacional de Ingreso.

Cada uno de los promedios en cuestión puede ser calculado por los usuarios, y es posible constatar si realmente se trata del usado para el cálculo del índice, pero después deben ser comparados con el grupo que conforman los egresados de ese instituto de EM, de manera que, si el bachiller en cuestión se graduó junto con 400 aspirantes, deberá conocer el promedio de cada uno de ellos para ordenarlos de menor a mayor y determinar el valor de la mediana del grupo, esa comparación va a producir el $19.5 \%$ del índice y el candidato no está en capacidad de calcularlo, no se dice cómo esta diferencia entre el promedio del candidato y la mediana del grupo se convertirá en el $19.5 \%$ de su índice, cuál es el algoritmo matemático que permite que la diferencia entre las puntuaciones en cada candidato llegue a representar el $19.5 \%$ de su índice. Similar situación observamos con el promedio especifico, el usuario recibe una tabla en la que se indica cómo calcularlo y él lo puede hacer, pero puede llegar hasta allí, lo cual significa otro $19.5 \%$ que no puede auditar. En total el 39\% de la información que constituye el índice multivariado es inauditable. El único que sabe si los cálculos están bien hechos es la persona que los hizo, por eso lo rechazamos. Es bueno, además, recalcar que el modelo no es tan multivariado como su nombre lo indica, dado que el $97.5 \%$ del índice se obtiene de las notas de cada aspirante.

En relación con el $2.50 \%$ correspondiente a otras variables, los bachilleres recién graduados solo podrán obtener un $0.30 \%$, que corresponde a quienes solicitan carreras dentro de su región, es decir, que para ellos el tope de su índice seria $97.80 \%$, no recibirían el porcentaje que corresponde a la participación en procesos anteriores ni el que corresponde a la asignación previa de plazas. A pesar de todas las inconsistencias, este procedimiento de asignación estuvo vigente hasta el proceso de 2014. 


\section{La proposición aprobada por el CNU en diciembre del 2014}

El 16 de diciembre del 2014, en reunión ordinaria del CNU, el ministro formuló oralmente algunas proposiciones en relación a la admisión a la educación superior, la primera de ellas relativa a las variables que a partir de ese momento debían regir el sistema nacional de ingreso, que quiere decir: el promedio de notas que representaría el 50\% del nuevo índice, el nivel socioeconómico que debía aportar el 30\%, la territorialidad, antigua regionalización según lo expresado en el debate que se suscitó en el consejo, que debe aportar el 15\%, y el 5\% faltante que debe utilizarse para apreciar las labores comunitarias realizadas por los aspirantes. No se consignó un documento sobre la proposición, simplemente se indicó que se trataba de una proposición política y que luego se presentaría un documento. No se hizo alusión a evaluaciones sobre los procesos existentes que permitieran advertir la justificación de los cambios. En la discusión de esta proposición oral no se describió la operacionalización de las variables, por lo que no fue posible realizar una evaluación exhaustiva de la calidad y pertinencia de la misma, ni de los alcances o consecuencias de la proposición.

\section{Observaciones u objeciones a la proposición aprobada por el CNU:}

Se necesitaría conocer los estudios en los cuales se evaluó el proceso anterior para establecer si, con base en los resultados, se puede deducir que se justifica un cambio como el propuesto. No se objeta el promedio de notas como criterio de selección, que es el de mayor poder predictivo, sin embargo, nos preguntamos por qué se disminuye casi a la mitad su participación en el índice, pasando de $97.5 \%$ a solamente el $50 \%$ en la nueva versión. No podemos hablar sobre la auditabilidad del componente porque esto depende de la operacionalización que se haga de cada variable, en el modelo anterior pudimos observar que el 39\% de la información no era auditable.

En relación con el uso del nivel socioeconómico, conocemos que la OPSU dispone del cuestionario Graffar modificado en el cual se pregunta sobre una serie de aspectos, estas preguntas se acompañan con cinco opciones de respuesta numeradas del 1 al 5. Tendrán mayor puntaje quienes se encuentren en las peores condiciones socioeconómicas en este país. La información que produce el cuestionario no es comprobable a menos que podamos visitar los cuatrocientos cincuenta mil hogares, esta constituye un dato ilustrativo, no podemos declarar como criterio información que no está corroborada. La inclusión de este criterio evidencia la ligereza con que se elaboró la proposición al conocer que esta información constituirá el $30 \%$ del índice, los usuarios pueden contestar de manera que sus respuestas permitan aumentar su puntuación en lugar de suministrar la información que se les solicita.

De la discusión de los rectores en el CNU se desprende que se ha llamado territorialidad a lo que antes se llamó regionalización, el propósito es otorgar preferencia a quienes soliciten carreras universitarias en la entidad federal en la cual obtuvieron el título de bachiller, en este sentido, debemos indicar lo siguiente: si el aspirante solicita carreras como Educación o Administración es probable que pueda cursar la carrera cerca de su casa 
por la cantidad de opciones que estas carreras ofrecen en todo el país, pero si desea estudiar Biología Marina tendrá que irse a la isla de Margarita, si lo que quiere estudiar es Ingeniería Forestal tendrá que trasladarse a Mérida, independientemente de donde estudió la educación media, ¿̇a nombre de qué justicia podemos penalizar por estos últimos ejemplos a un aspirante proveniente del Táchira o Bolívar? Una situación parecida confrontaremos con las carreras de Medicina, Psicología o la carrera militar. Esta variable tampoco está operacionalizada, se desconoce cómo se asignan las puntuaciones.

Por último, se ofrece un 5\% del índice para considerar la participación del aspirante en labores comunitarias, estas no se especifican, sin embargo, por la información que se manejó en la reunión del 16 de diciembre del 2014, podríamos esperar cualquier cosa: consignar un video en el cual se ayude a un anciano a pasar una calle, o una foto donde aparezca revendiendo los productos alimenticios escasos en el barrio, y aun cuando los precios por alguna razón estén elevados, se aprecia su voluntad de acercarlos a la población.

\section{El proceso actual y su condición de inauditable}

Cualquier proceso de selección y admisión de aspirantes a la educación superior es de intervención social, ya que se están tomando decisiones que afectan y condicionan el futuro del individuo y el de su familia. La implantación de nuevas versiones ameritan realizar campañas divulgativas para dar a conocer a la comunidad cuáles son los procedimientos a seguir, cómo se desarrollará el proceso como tal, cuáles son las variables que se considerarán y la operacionalización de las mismas, cuáles son las categorías y qué peso específico tendrá cada una de ellas, es decir, la comunidad debe estar informada a detalle sobre todo el proceso a desarrollar. El candidato debe estar informado sobre cómo se va a usar la información que proporcione, de qué manera se utilizará cada uno de los recaudos que le solicitan. Las IES deben recibir información detallada de los asignados, y también de los no asignados, y estas listas deben permitir explicar por qué unos fueron asignados y otros no. Este proceso es inauditable por decisión del ministro de educación universitaria, entre otras cosas, porque no se ha ocupado de informar por escrito los detalles de este adefesio (UCV, 2015).

Lo que está escrito es tan poco que no permite hacer mayores apreciaciones, cuando han decidido informar algo al respecto, lo que nos encontramos son contradicciones, el ministro, al ofrecer las listas de asignados, dice que, tal cual lo "acordado" en la reunión del CNU de diciembre del 2014 , las cuatro variables consideradas para la elaboración de las listas fueron: promedio de notas de bachillerato $50 \%$, condiciones socioeconómicas $30 \%$, territorialidad $15 \%$ y participación social $5 \%$. Estas constituyen macro variables y no se indican las variables en las que ellas se descomponen, ni cuáles son las categorías de cada una, mucho menos cuáles son los pesos específicos. Con base en esta información básica, se construyeron las siguientes tablas, en ellas se calcula un índice que alcanza un tope de cien, en el cual se asigna el máximo valor a las variables de territorialidad y participación social por desconocer totalmente su definición y operacionalización (González, 2015). 
Figura 5

\begin{tabular}{|c|c|c|c|c|c|c|c|c|c|c|c|c|c|c|c|c|c|c|c|}
\hline & \multirow[b]{3}{*}{ Puntaje NSE } & \multicolumn{17}{|c|}{ Nivel socioeconómico (30\%) } & \multirow{3}{*}{$\begin{array}{c}\text { Otras V } \\
(20 \%)\end{array}$} \\
\hline & & \multicolumn{3}{|c|}{$A$} & \multicolumn{3}{|c|}{ B } & \multicolumn{3}{|c|}{$\mathrm{C}$} & \multicolumn{4}{|c|}{$\mathrm{D}$} & \multicolumn{4}{|c|}{$E$} & \\
\hline & & 4 & 5 & 6 & 7 & 8 & 9 & 10 & 11 & 12 & 13 & 14 & 15 & 16 & 17 & 18 & 19 & 20 & \\
\hline PNotasB (50\%) & Contribución & 6 & 7.5 & 9 & 10.5 & 12 & 13.5 & 15 & 16.5 & 18 & 19.5 & 21 & 22.5 & 24 & 25.5 & 27 & 28.5 & 30 & 20 \\
\hline 20 & 50 & 56 & 57.5 & 59 & 60.5 & 62 & 63.5 & 65 & 66.5 & 68 & 69.5 & 71 & 72.5 & 74 & 75.5 & 77 & 78.5 & 80 & 20 \\
\hline 19 & 47.5 & 53.5 & 55 & 56.5 & 58 & 59.5 & 61 & 62.5 & 64 & 65.5 & 67 & 68.5 & 70 & 71.5 & 73 & 74.5 & 76 & 77.5 & 20 \\
\hline 17 & 42.5 & 48.5 & 50 & 51.5 & 53 & 54.5 & 56 & 57.5 & 59 & 60.5 & 62 & 63.5 & 65 & 66.5 & 68 & 69.5 & 71 & 72 & 20 \\
\hline 16 & 40 & 46 & 47.5 & 49 & 50.5 & 52 & 53.5 & 55 & 56.5 & 58 & 59.5 & 61 & 62.5 & 64 & 65.5 & 67 & 68.5 & 70 & 20 \\
\hline 15 & 37.5 & 43.5 & 45 & 46.5 & 48 & 49.5 & 51 & 52.5 & 54 & 55.5 & 57 & 58.5 & 60 & 61.5 & 63 & 64.5 & 66 & 67.5 & 20 \\
\hline 14 & 35 & 41 & 42.5 & 44 & 45.5 & 47 & 48.5 & 50 & 51.5 & 53 & 54.5 & 56 & 57.5 & 59 & 60.5 & 62 & 63.5 & 65 & 20 \\
\hline 13 & 32.5 & 38.5 & 40 & 41.5 & 43 & 44.5 & 46 & 47.5 & 49 & 50.5 & 52 & 53.5 & 55 & 56.5 & 58 & 59.5 & 61 & 62.5 & 20 \\
\hline
\end{tabular}

En esta tabla se muestran las contribuciones de las variables consideradas según la puntuación alcanzada en cada una. En la primera y segunda columna se muestran los promedios de calificaciones de educación media que pudieran exhibir los bachilleres, desde el 20 hasta el 10, y a un lado la puntuación que aportaría al índice del que se gradúe con estas calificaciones, como el PNB aporta el 50\%, multiplicamos el promedio de notas por 2.5 y el 20 aportaría 50 puntos al índice. En la primera fila disponemos la información del nivel socioeconómico, en la segunda aparecen las categorías y en la tercera los puntos que corresponden a cada categoría, el puntaje se establece con las cuatro preguntas establecidas en la técnica Graffar modificada en opsu, los valores posibles van desde 4 hasta 20 y como la proporción de la variable es el 30\%, los aportes se consiguen multiplicando la puntuación por 1,5; quien obtenga una puntuación de 20 estará en las peores circunstancias o condiciones y su puntuación aportara 30 puntos al índice. En la última columna, otras variables (territorialidad y participación social) aportan $20 \%$ al índice, es decir, 20 puntos en nuestro ejemplo, y como desconocemos qué variables comprenden esta macro variable, le asignamos los 20 puntos a todos para medir la incidencia de las dos primeras.

En la siguiente tabla se muestran los resultados, los índices que se obtendrían en las diferentes situaciones, por ejemplo: un bachiller con 20 puntos de PNB obtendría 50 puntos por su promedio, más los 20 de las otras variables llegaría a 70 , y si se encuentra en las peores condiciones socioeconómicas esto le aportaría 30 puntos a su índice, que sería 100, primera casilla de la última columna. Si este candidato se encuentra en las mejores condiciones socioeconómicas responderá 1 en las cuatro preguntas y su puntuación será 4, que al multiplicar por 1.5 la variable aportaría 6 puntos y su índice sería de 76 en la primera casilla de la segunda fila. Este índice sería igual al que obtendría un candidato con 11 puntos de PNB y una condición socioeconómica de categoría $\mathrm{E}$, de manera que el procedimiento prácticamente anula la importancia que pueda tener el promedio de notas, que es el mejor predictor del éxito académico utilizado en los criterios de ordenamiento, y lo hace precisamente por una información que la opsu da por buena porque nunca se comprueba la veracidad de la situación socioeconómica que declara el aspirante, ya que los hogares no son visitados para corroborar la información suministrada.

Cuando se recibieron las listas de aspirantes asignados en las universidades, al pie de cada página aparecía una nota que decía "Las variables tomadas en cuenta para la definición de índice académico son las siguientes: regionalización, área del conoci- 
miento, asignación previa, participación previa, promedio de notas, promedio global e índice de dispersión global" (CNU, 2015). La información inicial otorgada por el ministro en la reunión del CNU hablaba de cuatro variables utilizadas en la simulación anterior al consignar las listas de asignados al pie de cada hoja, aparece esta lista de siete variables y solo coinciden en el promedio de notas, no se explica el contenido de ninguna de ellas y por supuesto tampoco dice cuáles son las categorías ni los pesos correspondientes para cada una.

Figura 6. Índice de asignación

\begin{tabular}{|c|c|c|c|c|c|c|c|c|c|c|c|c|c|c|c|c|c|}
\hline \multirow{3}{*}{$\begin{array}{c}\text { Promedio } \\
\text { NotasB } \\
20\end{array}$} & \multicolumn{17}{|c|}{ Categorías del nivel socioeconómico } \\
\hline & \multicolumn{3}{|c|}{$A$} & \multicolumn{3}{|c|}{ B } & \multicolumn{3}{|c|}{ C } & \multicolumn{4}{|c|}{$\mathrm{D}$} & \multicolumn{4}{|c|}{$\mathrm{E}$} \\
\hline & 76 & 77.5 & 79 & 80.5 & 82 & 83.5 & 85 & 86.5 & 88 & 89.5 & 91 & 92.5 & 94 & 95.5 & 97 & 98.5 & 100 \\
\hline 19 & 73.5 & 75 & 76.5 & 78 & 79.5 & 81 & 82.5 & 84 & 85.5 & 87 & 88.5 & 90 & 91.5 & 93 & 94.5 & 96 & 97.5 \\
\hline 18 & 71 & 72.5 & 74 & 75.5 & 77 & 78.5 & 80 & 81.5 & 83 & 84.5 & 86 & 87.5 & 89 & 90.5 & 92 & 93.5 & 95 \\
\hline 17 & 68.5 & 70 & 71.5 & 73 & 74.5 & 76 & 77.5 & 79 & 80.5 & 82 & 83.5 & 85 & 86.5 & 88 & 89.5 & 91 & 92.5 \\
\hline 16 & 66 & 67.5 & 69 & 70.5 & 72 & 73.5 & 75 & 76.5 & 78 & 79.5 & 81 & 82.5 & 84 & 85.5 & 87 & 88.5 & 90 \\
\hline 15 & 63.5 & 65 & 66.5 & 68 & 69.5 & 71 & 72.5 & 74 & 75.5 & 77 & 78.5 & 80 & 81.5 & 83 & 84.5 & 86 & 87.5 \\
\hline 14 & 61 & 62.5 & 64 & 65.5 & 67 & 68.5 & 70 & 71.5 & 73 & 74.5 & 76 & 77.5 & 79 & 80.5 & 82 & 83.5 & 85 \\
\hline 13 & 58.5 & 60 & 61.5 & 63 & 64.5 & 66 & 67.5 & 69 & 70.5 & 72 & 73.5 & 75 & 76.5 & 78 & 79.5 & 81 & 82.5 \\
\hline 12 & 56 & 57.5 & 59 & 60.5 & 62 & 63.5 & 65 & 66.5 & 68 & 69.5 & 71 & 72.5 & 74 & 75.5 & 77 & 78.5 & 80 \\
\hline 11 & 53.5 & 55 & 56.5 & 58 & 59.5 & 61 & 62.5 & 64 & 65.5 & 67 & 68.5 & 70 & 71.5 & 73 & 74.5 & 76 & 77.5 \\
\hline 10 & 51 & 52.5 & 54 & 55.5 & 57 & 58.5 & 60 & 61.5 & 63 & 64.5 & 66 & 67.5 & 69 & 70.5 & 72 & 73.5 & 75 \\
\hline
\end{tabular}

No se puede auditar lo que no se conoce, y este proceso está convertido en una caja negra. Desconocemos el procedimiento general y también los específicos, no se sabe cuál es el algoritmo que permite construir el índice de asignación. Estamos en presencia de un proceso de intervención social que debía ser conocido a detalle por la comunidad, y el gobierno nos lo presenta cubierto por la mayor opacidad posible, podemos suponer a partir del interés de que no se sepa cómo lo hicieron.

El otro aspecto a considerar dentro del proceso es lo perverso que resulta para los aspirantes, y para el país, haber usado como criterio de ordenamiento información no verificada, proveniente de la aplicación del cuestionario que sirve de base para la clasificación de poblaciones a través de la Técnica Graffar. Los aspirantes respondieron el cuestionario durante el proceso de registro en el Proceso Nacional de Ingreso, la OPSU debió visitar los 450.000 hogares para verificar la certeza de la información recibida si su decisión era utilizarla para el ordenamiento de los candidatos, de manera que los aspirantes fueron asignados o rechazados con base en una información que la OPSU "cree" que es verdad. Cuál es el nivel de seguridad con el que se toman decisiones cuando lo hacemos sobre informaciones no verificadas. No hay manera de verificar la información de las variables no académicas que no sea la visita de los 450 mil hogares con personal calificado. 
Para proponer en el área de admisión hay que conocer la naturaleza del proceso y valorar sus repercusiones sociales y económicas

Para realizar un buen trabajo en el área de admisión es necesario reconocer que el proceso es bastante complejo y que en él están presentes un número importante de variables y atributos que deben ser considerados, y que, aun cuando no aparezcan declarados en el modelo, tendrán una participación importante.

En este conjunto de variables hay un grupo constituido por las académicas: el promedio de notas de educación media, la aptitud académica medida a través del desarrollo de los lenguajes, la habilidad para captar relaciones espaciales o los conocimientos específicos de las asignaturas; estas son las variables duras que se utilizan como criterio de selección, se escogen las que sean factibles de medir de una forma precisa y demostrable (Sarco, 2003). Sobre los datos que aportan debemos tener la capacidad para realizar demostraciones de archivo.

Figura 7

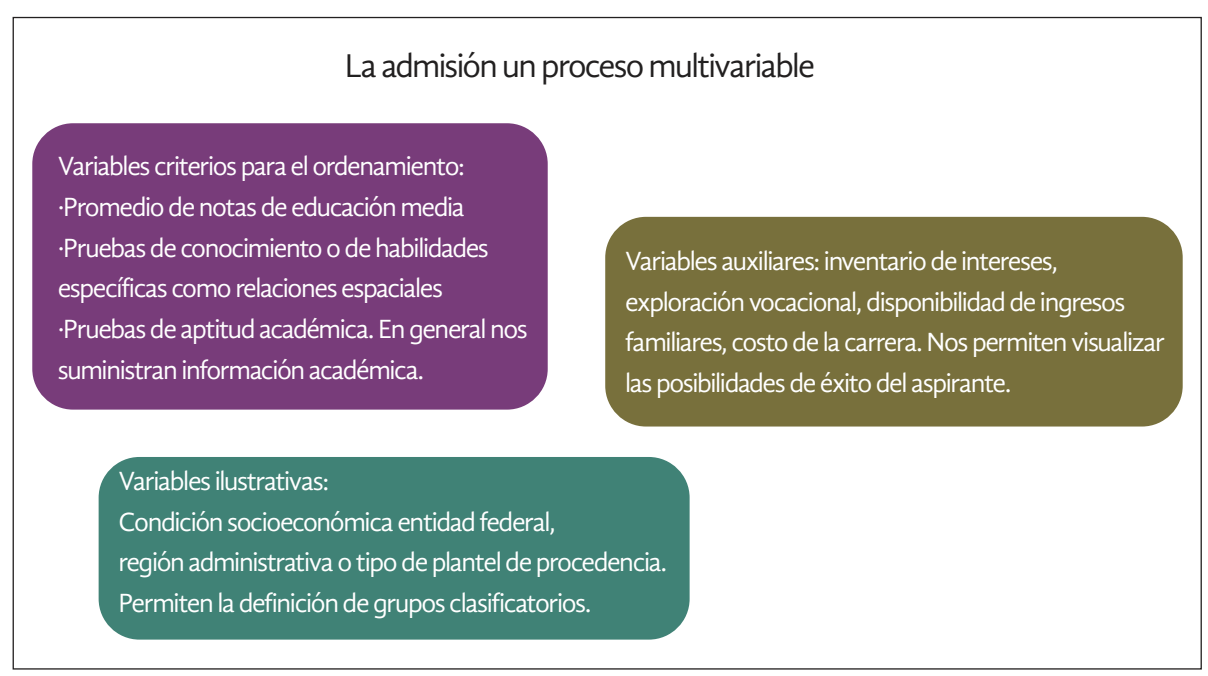

El segundo grupo está compuesto por variables auxiliares pero con gran importancia en la toma de decisiones: la exploración vocacional, los requisitos propios de las carreras, la información sobre las oportunidades de estudio, el conocimiento sobre los proyectos familiares, el respaldo económico que pueda brindar la familia, etc. Conocer en qué área del conocimiento puede el individuo lograr un desarrollo pleno de sus potencialidades, o saber si la familia puede costear los estudios, son cosas que resultan de un interés capital para garantizar el éxito en la carrera. El tercer grupo de variables son las ilustrativas, se utilizan para evaluar los procesos y ayudar en la definición de políticas sociales o económicas que se deben implementar en los casos donde haya iniquidad en el tratamiento de alguno de los grupos de aspirantes, aquí se encuentran las variables socioeconómicas, el nivel educativo de la madre, las condiciones de alojamiento de la vivienda, 
la información sobre ubicación geográfica y la dependencia administrativa del plantel de procedencia. Algunas de ellas están fuertemente asociadas al éxito en los estudios pero no deben ser usadas como criterio de selección, supongamos que se revisan las listas de graduados en una universidad durante el último quinquenio, y se observa que el $60 \%$ de ellos tiene una madre con nivel universitario, esta es una información muy importante para un político, que deberá establecer cuáles son las características resaltantes de los hogares con madres universitarias para generar políticas sociales que contribuyan a homologar las posibilidades de los aspirantes con madres no universitarias, como podría ser extender la red de bibliotecas o generar grupos de asesoría académica en los centros de estudios; lo que no podemos hacer, porque resultaría absurdo, es decidir que, dado que se gradúan más los hijos de madres universitarias, eso se va a establecer como un criterio de selección porque está asociado al éxito. Cada una de las variables juega un papel importante, y su clasificación en los grupos depende de donde resalte su contribución, aspecto que debemos tener en cuenta para evaluar cualquier proposición relativa a la admisión universitaria. Las variables deben ser usadas donde su información resulte más provechosa al aspirante.

La oferta de los núcleos de vicerrectores académicos y de secretarios del CNU como solución real del problema en que se ha convertido la admisión a la educación superior

Los núcleos de vicerrectores académicos y de secretarios del CNU produjeron varios documentos con recomendaciones de procesos alternativos que pudieran mejorar el diseño puesto en práctica a partir del 2008 (Ríos, 2010) en el último documento consignado ante la OPSU en 2015. El "Sistema Nacional de Ingreso a la Educación Superior" se propone como un modelo en el cual ningún aspirante ingresa al subsistema sin una evaluación académica previa, de esta manera, ingresarían los que posean las competencias o cumplan con los requisitos establecidos. El Estado, a través de las IES, ofrecerá cursos de nivelación para los aspirantes que no satisfagan los requisitos, una vez finalizada esta etapa asistencial, los candidatos se someterán de nuevo a las pruebas, aquellos que hayan logrado las competencias necesarias ingresarían a las carreras solicitadas, y los que no hayan superado aún su situación serán remitidos a la OPSU para una reorientación de carreras, de esta manera, a ningún aspirante se le niega el cupo o la posibilidad de obtenerlo, se le ofrece una etapa de nivelación y solo en caso de no lograr satisfacer los requisitos de ingreso deberá reconsiderar su selección de carreras.

Esta proposición fue remitida por los coordinadores de ambos núcleos en enero de 2015 para el director de la OPSU y para el ministro de educación universitaria, acompañada de los siguientes documentos adicionales:

- Lista de los documentos elevados ante las autoridades del Ministerio del Poder Popular para la Educación Universitaria.

- Elementos conceptuales que orientan el modelo propuesto en 2010, Calidad, Inclusión y Equidad. 
- Características del modelo: cobertura nacional, incorpora el uso de las Técnicas de Información y Comunicación, es único y permanente.

- Ventajas de su implementación: para el aspirante, para las instituciones de educación superior, para la OPSU.

- Lista de compromisos de los entes involucrados y de los resultados esperados.

\section{Referencias}

CNU-OPSU (1973). Actas de las Reuniones realizadas en el año.

CNU-OPSU (2015). Lista de Asignados a la UCV.

CNU-OPSU (2009). Nuevo Sistema Nacional de Ingreso y Prosecución en la Educación Superior Venezolana. Cuadernos OPSU. No.10

González, Jesús (2008). Asignación de aspirantes a través del Sistema Nacional de Ingreso a la Educación Superior SINIES. Caracas, UCV.

González, Jesús. Asignación de aspirantes a través del Sistema Nacional de Ingreso a la Educación Superior SINIES (2015). Caracas, UCV.

Menéndez, Omar (2000). El valor predictivo de la Prueba de Aptitud Académica. Caracas, OPSU. Mimeografiado.

Ríos, Pablo (coordinador) (2010). Sistema de Ingreso a la Educación Superior. Propuesta de la Comisión designada por las Juntas Directivas del Núcleo de Vicerrectores Académicos y el Núcleo de Secretarios a ser presentada en el CNU.

Rojas, M. (2008). Modelo Multivariado basado en Lógica Difusa para el Proceso de Asignación de Cupos del CNU. Documento mimeografiado, OPSU, Caracas.

Sarco, A. y Bonucci, M. (1994) La Política Nacional de Admisión a la Educación Superior en Venezuela. Conferencia en I Encuentro de Secretarios de Universidades de América Latina y el Caribe. Revista Análisis, volumen 3, $\mathrm{N}^{\circ} 1$, Caracas.

Sarco, Amalio (2003, Junio). El Proceso Nacional de Admisión. I Jornadas de Orientación. OPSU-UCV, Caracas, Venezuela.

Sarco, A. y Fuenmayor, L. (2004). Evaluación de los nuevos procedimientos utilizados en la fase de asignación del Proceso Nacional de Admisión. Experiencia universitaria-Revista Venezolana de la Educación Superior, Vol. 2, (Nº3), pp: 35-69 (OPSU-CNU-MES).

UCV, Vicerrectorado Académico (2015). Comisión ad-hoc para el análisis del Proceso de Asignación de aspirantes a través de CNU. Informe de Resultados. Mimeografiado.

UNESCO (2009). Conferencia Mundial sobre la Educación Superior 2009: La nueva dinámica de la educación superior y la investigación para el cambio social y el desarrollo. Comunicado. París, 5-8 de julio de 2009. 


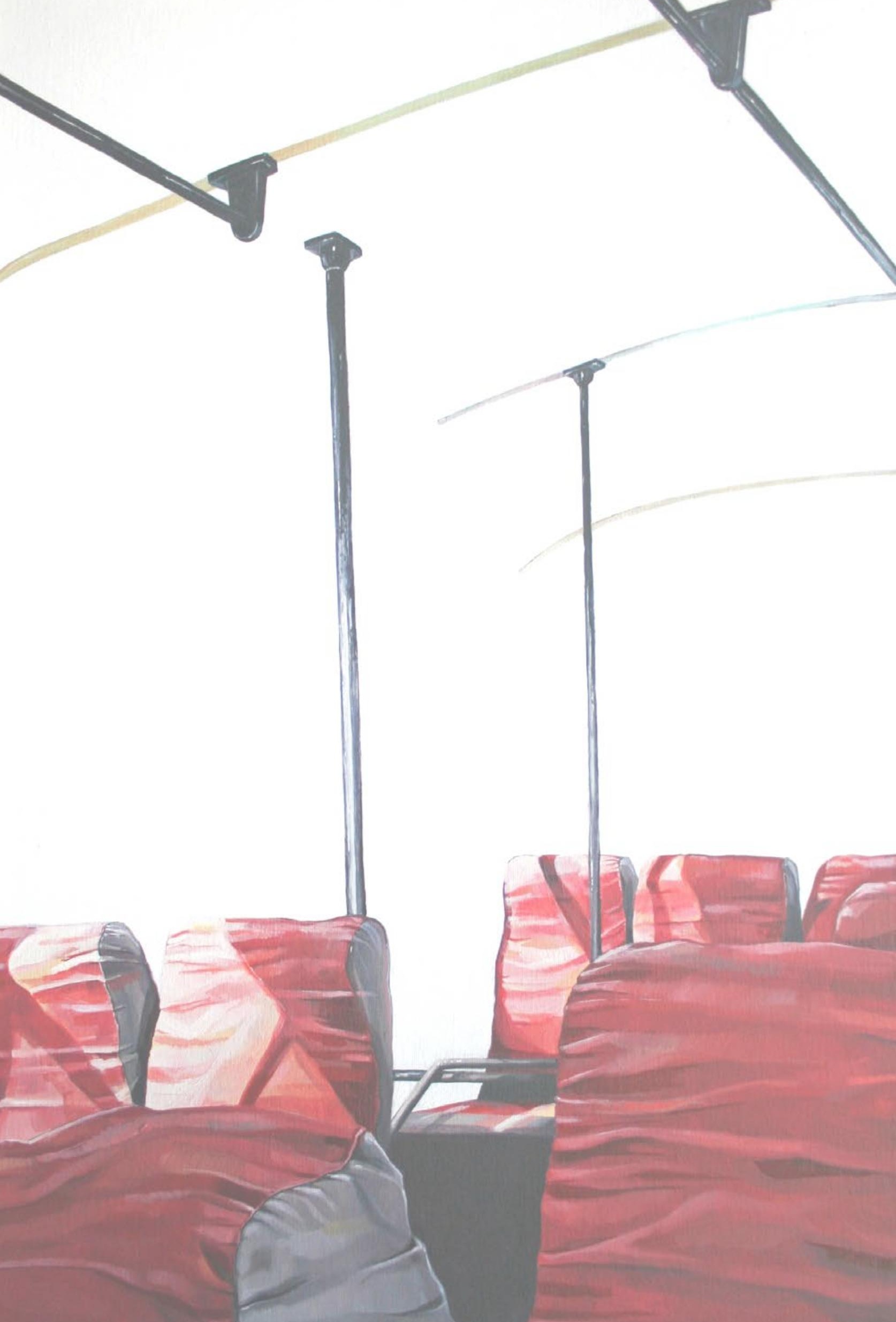

